\title{
Regulation of Lignin Biosynthesis by Post-translational Protein Modifications
}

\author{
Daniel B. Sulis and Jack P. Wang* \\ Forest Biotechnology Group, Department of Forestry and Environmental Resources, North Carolina State University, Raleigh, \\ NC, United States
}

OPEN ACCESS

Edited by:

Jaime Barros-Rios,

University of North Texas,

United States

Reviewed by:

Chang-Jun Liu,

Brookhaven National Laboratory

(DOE), United States Jeongim Kim,

University of Florida, United States Xiaolan Rao,

University of North Texas,

United States

*Correspondence:

Jack P. Wang

jpwang@ncsu.edu

Specialty section:

This article was submitted to

Plant Biotechnology,

a section of the journal

Frontiers in Plant Science

Received: 21 March 2020

Accepted: 04 June 2020

Published: 02 July 2020

Citation:

Sulis DB and Wang JP (2020) Regulation of Lignin Biosynthesis by

Post-translational Protein

Modifications.

Front. Plant Sci. 11:914.

doi: 10.3389/fpls.2020.00914
Post-translational modification of proteins exerts essential roles in many biological processes in plants. The function of these chemical modifications has been extensively characterized in many physiological processes, but how these modifications regulate lignin biosynthesis for wood formation remained largely unknown. Over the past decade, post-translational modification of several proteins has been associated with lignification. Phosphorylation, ubiquitination, glycosylation, and S-nitrosylation of transcription factors, monolignol enzymes, and peroxidases were shown to have primordial roles in the regulation of lignin biosynthesis. The main discoveries of post-translational modifications in lignin biosynthesis are discussed in this review.

Keywords: lignin, lignification, proteins, PTMs, SCW, trees, wood formation

\section{INTRODUCTION}

Wood is a biological composite and a valuable source of feedstock for construction timber, pulp and paper, and biofuels (Wang et al., 2018). Wood is composed of secondary xylem tissue formed by secondary cell walls (SCW) (Fromm, 2013). The secondary xylem consists of two types of cells: fibers, which provide mechanical support, and tracheary elements composed of vessels (not found in gymnosperm wood) and tracheids (found in both angiosperm and gymnosperm) for water and solutes transport (Demura and Fukuda, 2007). SCW is mainly composed of cellulose, hemicelluloses, and lignin, in different ratios of these constituents (Li et al., 2011; Ye and Zhong, 2015).

Lignin is a phenolic polymer formed by phenylpropanoid monomeric units, 4-coumaryl alcohol (H-unit), coniferyl alcohol (G-unit), and sinapyl alcohol (S-unit), also known as monolignols. The biosynthesis of lignin occurs in several consecutive reactions involving up to 11 different enzyme families and 24 metabolites (Wang et al., 2019b). The pathway is complex and regulated by a network of substrates and inhibitors in the conversion of phenylalanine or tyrosine to monolignols (Wang et al., 2014). The monolignols are then transported to the lignifying zone and oxidized by peroxidases and laccases to phenoxy radicals (Li et al., 2014). Many efforts have been made to elucidate the regulation of the lignin biosynthetic pathway. Transcription factors (TFs) associated with lignification have been identified for Pinus (Patzlaff et al., 2003a,b), Eucalyptus (Goicoechea et al., 2005; Legay et al., 2010), and Populus (Karpinska et al., 2004; Zhong et al., 2010b; Ohtani et al., 2011; Li et al., 2012, 2015; Tian et al., 2013; Wang S. et al., 2015; Yang et al., 2017; Chen et al., 2019; Gui et al., 2019; Zheng et al., 2019) tree species. These TFs impart transactivation and transrepression of monolignol genes and other TFs in complex hierarchical transcriptional regulatory networks. Some TFs associated with monolignol biosynthesis form protein-protein 
(TF-TF) interactions that may affect TF-target DNA binding (Chen et al., 2019). Lignification is also regulated at the enzyme level. Monolignol biosynthetic enzymes can directly interact with each other (e.g., Ptr4CL3-Ptr4CL5, PtrC3HPtrC4H, At4CL1-AtC3H, At4CL1-AtC4H, and AtCCR1-AtC4H interactions) (Chen et al., 2011; Chen H. -C. et al., 2014; Gou et al., 2018; Wang et al., 2019a) or indirectly through common mediators such as AtMSPBs (e.g., AtC3H-AtMSPBs, AtC4HAtMSPBs, AtF5H-AtMSPBs interactions) (Gou et al., 2018). In addition, monolignol biosynthetic enzymes can interact with proteins in other biological pathways (e.g., OsCCR1-OsRac1, ZmCCoAOMT/ZmHCT-ZmRp1 interactions) (Kawasaki et al., 2006; Wang G. H. et al., 2015; Wang and Balint-Kurti, 2016). These interactions may modulate enzyme stability, activity, and metabolic flux in lignin biosynthesis, increase the biosynthesis of lignin in response to pathogen infection, and suppress plant hypersensitive response (Kawasaki et al., 2006; Chen et al., 2011; Chen H. -C. et al., 2014; Wang G. H. et al., 2015; Wang et al., 2019a; Wang and Balint-Kurti, 2016; Gou et al., 2018). However, the role of post-translational modifications (PTMs) in SCW formation remains poorly understood.

PTMs are covalent processes that alter the properties of proteins by proteolytic cleavage or addition of modifying groups to one or more amino acids (Mann and Jensen, 2003). Over 200 different types of PTMs have been identified, ranging from small chemical modifications (e.g., phosphorylation and acetylation) to the addition of complete proteins (e.g., ubiquitination) (Beltrao et al., 2013; Spoel, 2018). PTMs are essential for many proteins and can affect the localization, stability, structure, activity, and molecular interactions of proteins (Nørregaard Jensen, 2004; Wang J. P. et al., 2015). In plants, PTMs are associated with plant growth and development, biotic and abiotic stress response, and metabolism (Catala et al., 2007; Mazzucotelli et al., 2008; Stulemeijer and Joosten, 2008; Miura and Hasegawa, 2010; Friso and van Wijk, 2015). Phosphorylation of cellulose synthases and cellulose synthase-like proteins were identified to have essential roles in regulating the activity and distribution of cellulose synthase complexes (CSCs) along microtubules (Speicher et al., 2018). The ubiquity of PTMs and their diverse regulatory functions is indicative of the complexity of secondary cell wall biosynthesis in general, and lignin biosynthesis in particular (Table 1).

\section{PHOSPHORYLATION OF MONOLIGNOL BIOSYNTHETIC ENZYMES}

Lignin biosynthesis is typically perceived as a matrix of linear or branched enzymatic reactions that successively modify the aromatic ring of the phenylpropane units and conversion of the side-chain carboxyl to an alcohol moiety. The involvement of many enzymes suggests that the pathway is well coordinated to mediate precise control of the rate and ratios of monolignol biosynthesis for polymerization. PTMs provide an efficient way to impart spatiotemporal activation and inactivation of monolignol enzyme activities in plants. However, the identification of PTMs involved in lignin biosynthesis remains challenging. Some studies based on phosphoproteomics could not reliably detect phosphopeptides for monolignol enzymes in wood forming cells (Mauriat et al., 2015). The difficulty of detecting protein phosphorylation is in part due to the highly dynamic nature of the regulatory mechanism.

\section{PAL PHOSPHORYLATION}

Phenylalanine ammonia-lyase (PAL) is a family of enzymes that catalyzes the deamination of phenylalanine to cinnamic acid, representing the first reaction step in the phenylpropanoid pathway (Shi et al., 2013; Zhang and Liu, 2015). PAL enzymes have been extensively characterized for their protein structure, functionality, and primordial role in wood formation (Ritter and Schulz, 2004; Shi et al., 2013). Some evidence regarding the chemical modification of PAL has been proposed. In Phaseolus vulgaris (French Bean) suspension-cultured cells, a phosphopeptide derived from PAL2 was detected (Allwood et al., 1999). The authors suggested that the kinase responsible for the PAL2 phosphorylation belongs to the calmodulinlike domain protein kinase (CDPK) family (Allwood et al., 1999). Consistently, a recombinant PAL2 protein from $P$. trichocarpa $\times P$. deltoides was phosphorylated by CDPK derived from French bean suspensions and Arabidopsis (Allwood et al., 1999; Cheng et al., 2001). In PAL of Phyllostachys edulis, in silico functional analysis indicated a likely presence of nine casein kinase phosphorylation sites, eight protein kinase $\mathrm{C}$ phosphorylation sites, and two N-glycosylation sites (Gao et al., 2012). The high degree of PAL peptide sequence similarity across plant species and their conserved phosphorylation by CDPK may suggest a common phosphoregulatory mechanism for PAL in phenylpropanoid biosynthesis (Allwood et al., 1999; Cheng et al., 2001; Zhang and Liu, 2015).

The functional role of post-translational phosphorylation in PAL remains to be clarified. Allwood et al. (1999) showed that one hour after co-incubation of PAL2 and CDPK, the Km and $\mathrm{V}_{\text {max }}$ of PAL2 is slightly reduced. On the other hand, longer co-incubation of up to $4 \mathrm{~h}$ reduced the protein stability of the phosphorylated isoform of PAL2 and result in a 3-fold reduction in the $\mathrm{V}_{\max }$ when compared to the non-phosphorylated isoform (Allwood et al., 1999). The phosphorylation of PAL2 likely limits the rate of phenylalanine conversion to cinnamic acid, thereby influencing the metabolic flux for lignification.

The CDPK family of kinases has been associated with the regulation of biological processes encompassing plant growth, development, and response to biotic and abiotic stresses (Romeis, 2001; Schulz et al., 2013). Pathogen infections induce the expression and activity of PAL (Jones, 1984; Shoresh and Harman, 2008), which in turn promotes immune response mediated by salicylic acid production, enhanced physical barriers (lignin), and accumulation of antimicrobial compounds (e.g., phytoalexins) (Jones, 1984; Solecka, 1997; Chen et al., 2009; Adams-Phillips et al., 2010; Hamann, 2012; Yan and Dong, 2014). Since PAL transcript expression is upregulated under biotic stress, the CDPK-mediated phosphorylation of PAL may mark it for turnover, thus reducing PAL activity 
TABLE 1 | PTMs of proteins involved in lignification.

\begin{tabular}{|c|c|c|c|c|c|}
\hline Protein & Organism & Types of PTM & Detection of PTM & Effect of PTM & References \\
\hline PAL2 & P. vulgaris (French bean) & Phosphorylation & In vitro & Unknown & Allwood et al., 1999 \\
\hline PAL2 & P. trichocarpa $\times$ P. deltoides & Phosphorylation & In vitro & $\begin{array}{l}\text { Phosphorylation of PAL2 decreases } \mathrm{Km} \\
\text { and } \mathrm{V}_{\max }\end{array}$ & $\begin{array}{l}\text { Allwood et al., 1999; Cheng } \\
\text { et al., } 2001\end{array}$ \\
\hline AldOMT2 & P. trichocarpa & Phosphorylation & In vitro & $\begin{array}{l}\text { Phosphorylations of AldOMT2 reduce } \\
\text { enzyme activity }\end{array}$ & Wang J. P. et al., 2015 \\
\hline MYB4 & P.taeda & Phosphorylation & In vitro & $\begin{array}{l}\text { Phosphorylation of MYB4 reduces the } \\
\text { MYB4-transactivation activity over the } \\
\text { lignin target genes }\end{array}$ & Morse et al., 2009 \\
\hline RAl1 & O. sativa & Phosphorylation & In vitro & $\begin{array}{l}\text { Phosphorylation activates RAl1 and } \\
\text { increases the expression of PAL1. }\end{array}$ & $\begin{array}{l}\text { Kawasaki et al., 2006; Kim } \\
\text { et al., 2012; Akamatsu et al., } \\
\text { 2013; Nasir et al., } 2018\end{array}$ \\
\hline LTF1 & P. trichocarpa & Phosphorylation & In vivo and In vitro & $\begin{array}{l}\text { Phosphorylation reduces the LTF1 } \\
\text { stability via } 26 S \text { proteasome and } \\
\text { reduces the LTF1-transrepression } \\
\text { activity over the lignin target genes }\end{array}$ & Gui et al., 2019 \\
\hline PAL1-4 & A. thaliana & Ubiquitination & In vivo & $\begin{array}{l}\text { Ubiquitination reduces the protein } \\
\text { stability via } 26 S \text { proteasome }\end{array}$ & Zhang et al., 2013 \\
\hline CCR & O. sativa & Ubiquitination & Untested & $\begin{array}{l}\text { Interaction of OsCCR with SCFOsFBK1 } \\
\text { reduces the OsCCR stability via } 26 S \\
\text { proteasome }\end{array}$ & Borah and Khurana, 2018 \\
\hline MYB156 & P. tomentosa & Ubiquitination & Untested & $\begin{array}{l}\text { Interaction of MYB156 with UBC34 } \\
\text { reduces the MYB156-transactivation } \\
\text { over the lignin genes possibly } \\
\text { decreasing the MYB156 stability via } \\
\text { 26S proteasome }\end{array}$ & Zheng et al., 2019 \\
\hline MYB221 & P. tomentosa & Ubiquitination & Untested & $\begin{array}{l}\text { Interaction of MYB221 with UBC34 } \\
\text { reduces the MYB221-transactivation } \\
\text { over the lignin genes possibly } \\
\text { decreasing the MYB221 stability via } \\
26 \text { S proteasome }\end{array}$ & Zheng et al., 2019 \\
\hline VND7 & A. thaliana & S-nitrosylation & In vitro & $\begin{array}{l}\text { S-nitrosylation of VND7 decreases the } \\
\text { VND7-transactvation activity over the } \\
\text { SCW genes }\end{array}$ & Kawabe et al., 2018 \\
\hline VND7 & A. thaliana & Ubiquitination & Untested & $\begin{array}{l}\text { VND7 protein accumulates upon } \\
\text { treatment with } \mathrm{MG}-132 \text { in transformed } \\
\text { tobacco BY-2 cells }\end{array}$ & Yamaguchi et al., 2008 \\
\hline PXP1-6 & P. trichocarpa & Glycosylation & In vivo & Unknown & Christensen et al., 1998 \\
\hline PRX & Z. elegans & Glycosylation & In vitro & $\begin{array}{l}\text { Glycosylations change the PRX } \\
\text { catalytic efficiencies. }\end{array}$ & $\begin{array}{l}\text { Gabaldón et al., 2005, 2006, } \\
2007\end{array}$ \\
\hline
\end{tabular}

(Allwood et al., 1999) to maintain homeostasis (Figure 1A). The phosphorylation of PAL has also been suggested to translocate the proteins to membranes, which may contribute to metabolic channeling in lignin biosynthesis (Allwood et al., 1999; Rasmussen and Dixon, 1999).

\section{AldOMT2 PHOSPHORYLATION}

5-Hydroxyconiferaldehyde $O$-methyltransferase 2 (AldOMT2) catalyzes the 3/5-methylation of caffeoyl- and 5-hydroxyferuloylcontaining acids, aldehydes, and alcohols for monolignol biosynthesis. In $P$. trichocarpa. PtrAldOMT2 is the highest transcribed gene in the monolignol biosynthetic pathway and the third-highest transcribed gene in stem differentiating xylem (SDX). The protein abundance of PtrAldOMT2 is also the highest of all monolignol enzymes, accounting for $5.9 \%$ of the
SDX proteome (Shuford et al., 2012; Lin et al., 2013). Given the abundance in transcripts and proteins of PtrAldOMT2, regulation of its activity by transcriptional control is energyintensive. In contrast, regulation of PtrAldOMT2 activity by protein phosphorylation removes the need to synthesize/degrade RNA and protein, thus providing a rapid and energetically efficient mode of regulation for O-methyltransferase activity (Wang J. P. et al., 2015). Phosphoproteomic analysis by mass-spectrometry in SDX revealed two phosphopeptides in PtrAldOMT2 that contain either a phosphoserine at peptide position-123 or position-125. Concurrent phosphorylation of both Serine123 and Serine125 was not detected in vivo. The phosphorylation of PtrAldOMT2 is mediated by kinases in the SDX protein extract and could be reversed by phosphatase treatment (Wang J. P. et al., 2015). Phosphorylation reduced the enzymatic activity of recombinant PtrAldOMT2 considerably, and this reduction in activity was not due to 


\section{A}

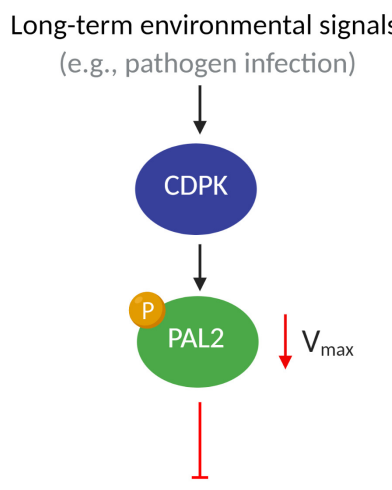

Lignification
B

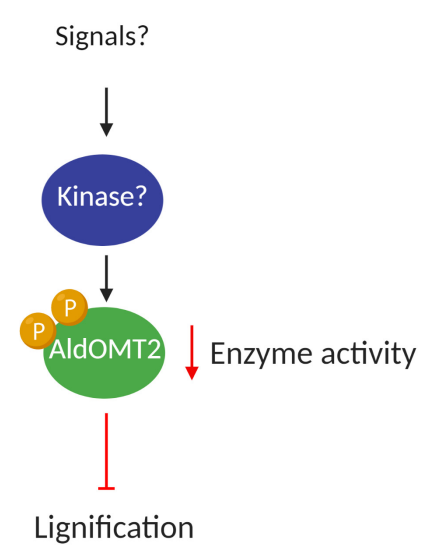

FIGURE 1 | A putative model of kinases regulating monolignol enzymes activity. (A) Under potential stimulus such as pathogen infections, the CDPK proteins phosphorylate PAL2, decreasing the $V_{\max }$ of the enzyme and reducing lignification in plants; (B) Kinase(s) protein(s), after an endogenous or environmental stimulus, phosphorylates AldOMT2 at either of the two phosphorylation sites and negatively regulates the AldOMT2 activity.

protein degradation (Wang J. P. et al., 2015). Moreover, site-directed mutagenesis by replacing either Serine123 or Serine125 with asparagine, a negatively charged amino acid that mimics the properties of phosphoserine, showed that Serine123 is more critical for phosphoregulation of enzyme activity. Serine123 is conserved in 43 of 46 (93\%) AldOMTs across diverse plant species (Wang J. P. et al., 2015), suggesting a conserved evolutionary phosphoregulation of the enzyme. The presence of two phosphoserine residues in PtrAldOMT2 and their distinct enzymatic regulations provide a basis for further exploring mechanistic insights to phosphoregulation in monolignol biosynthesis. Whether different kinases and signaling pathways regulate the two phosphorylation sites or if the sites are functionally redundant, remains to be determined (Wang J. P. et al., 2015). Lignification is regulated by many environmental and developmental stimuli that combinatorially modulate the rate and ratios of monolignol biosynthesis. The understanding of how PTMs transduce the regulation to changes in metabolic flux for monolignol biosynthesis would provide valuable knowledge of plant adaptation and cell wall biosynthesis (Figure 1B).

\section{PHOSPHORYLATION OF TFS IN LIGNIN BIOSYNTHESIS}

R2R3-MYBs are TFs that contain a DNA binding domain at the $\mathrm{N}$-terminus, which is composed of two imperfect helix-turn-helix repeats of approximately 50 amino acids (R2 and R3) (Jin and Martin, 1999; Patzlaff et al., 2003a). Protein members from the R2R3-MYB family of TFs are known to bind at AC rich ciselements present in the promoters of many genes in the lignin biosynthetic pathway (Lois et al., 1989; Joos and Hahlbrock, 1992; Leyva et al., 1992; Hauffe et al., 1993; Hatton et al., 1995; Logemann et al., 1995; Bell-Lelong et al., 1997; Séguin et al., 1997; Lacombe et al., 2000; Lauvergeat et al., 2002; Patzlaff et al., 2003a). Some R2R3-MYBs have been identified and characterized as key regulators of plant cell wall biosynthesis (Patzlaff et al., 2003a). PtMYB4 is an ortholog of AtMYB56 and AtMYB83 (Patzlaff et al., 2003a; Yang et al., 2016; Gui et al., 2019), which function as second layer transactivators of SCW biosynthesis in Arabidopsis (Yang et al., 2016). The overexpression of PtMYB4 in tobacco induced monolignol biosynthesis and promoted lignin accumulation in non-lignified cell types, suggesting that this TF regulates positively the lignin biosynthesis genes during the wood formation process (Patzlaff et al., 2003a).

Using a cDNA library from Pinus taeda SDX, Morse et al. (2009) screened MAPKs (PtMAPK6 and PtMAPK13) potentially involved in wood formation. In vitro phosphorylation assays demonstrated that PtMAPK6 could phosphorylate PtMYB1 and PtMYB4, transregulators of lignin biosynthesis in SDX. Replacement of Lysine68 for arginine abolished the kinase activity of PtMAPK6, demonstrating that the lysine is essential for mediating target phosphorylation. Serine236 was identified to be the phosphorylation site in PtMYB4. Replacement of Serine236 for a glutamate residue (mimicking constitutive phosphorylation of PtMYB4) did not interfere with the affinity for DNA binding but significantly reduced the transactivation of the target gene (Morse et al., 2009).

MAPKs are components of signal transduction networks that trigger a variety of biological processes in plants (Nühse et al., 2000). MAPK6 is associated with response to pathogen infection, ethylene response, and cell wall biosynthesis and remodeling (Andreasson and Ellis, 2010; Seifert and Blaukopf, 2010; Bacete et al., 2018). All these processes affect secondary cell wall biosynthesis for wood formation (Adams-Phillips et al., 2010; Fromm, 2013; Li et al., 2015; Ye and Zhong, 2015). In Oriza sativa, OsMAPK3 and OsMAPK6 are involved in the phosphorylation of OsRAI1 through a complex signaling cascade upon chitin elicitation. The activation of OsRAI1 by phosphorylation induces the transcript expression of defenseassociated genes including PAL1 (Kawasaki et al., 2006; Kim et al., 2012; Akamatsu et al., 2013; Nasir et al., 2018). In $P$. taeda, PtMAPK6 is expressed in cell differentiation stages 1, 2 (division and expansion), and 3, 4 (SCW synthesis) 
of SDX, but its kinase activity for PtMYB4 phosphorylation was mainly observed in the initial stages of 1 and 2 . The phosphorylated isoform of PtMAPK6, representing the active form of the enzyme, was only found in stages 1 and 2 (Morse et al., 2009). PtMAPK6 may be autophosphorylated or activated by other kinase-mediated phosphorylation during early xylogenesis. The activated PtMAPK6 then phosphorylates PtMYB4, reducing the TF transregulation of cell wall biosynthetic genes (Figure 2A). PtMAPK6 activity during early xylogenesis may function to suppress the expression of PtMYB4-regulated genes from resulting in premature deposition of lignin in dividing and expanding cells. The absence of PtMAPK6 activity in the late stages of differentiating xylem allows PtMYB4 to transactivate lignin biosynthetic genes in these cells (Morse et al., 2009).

LTF1 is the closest ortholog of AtMYB4 in P. deltoides $\times$ P. euramericana (Gui et al., 2019). Arabidopsis AtMYB4 mutants showed increased expression of $C 4 \mathrm{H}$ and reduced expression of CCOAOMT. Overexpression of AtMYB4 in tobacco increased the expression of CCOAOMT and reduced the expression of $C 4 H, 4 C L$, and CAD genes (Jin et al., 2000). In $P$. deltoides $\times P$. euramericana, LTF 1 is mainly expressed in the SDX as a transrepressor of monolignol biosynthetic

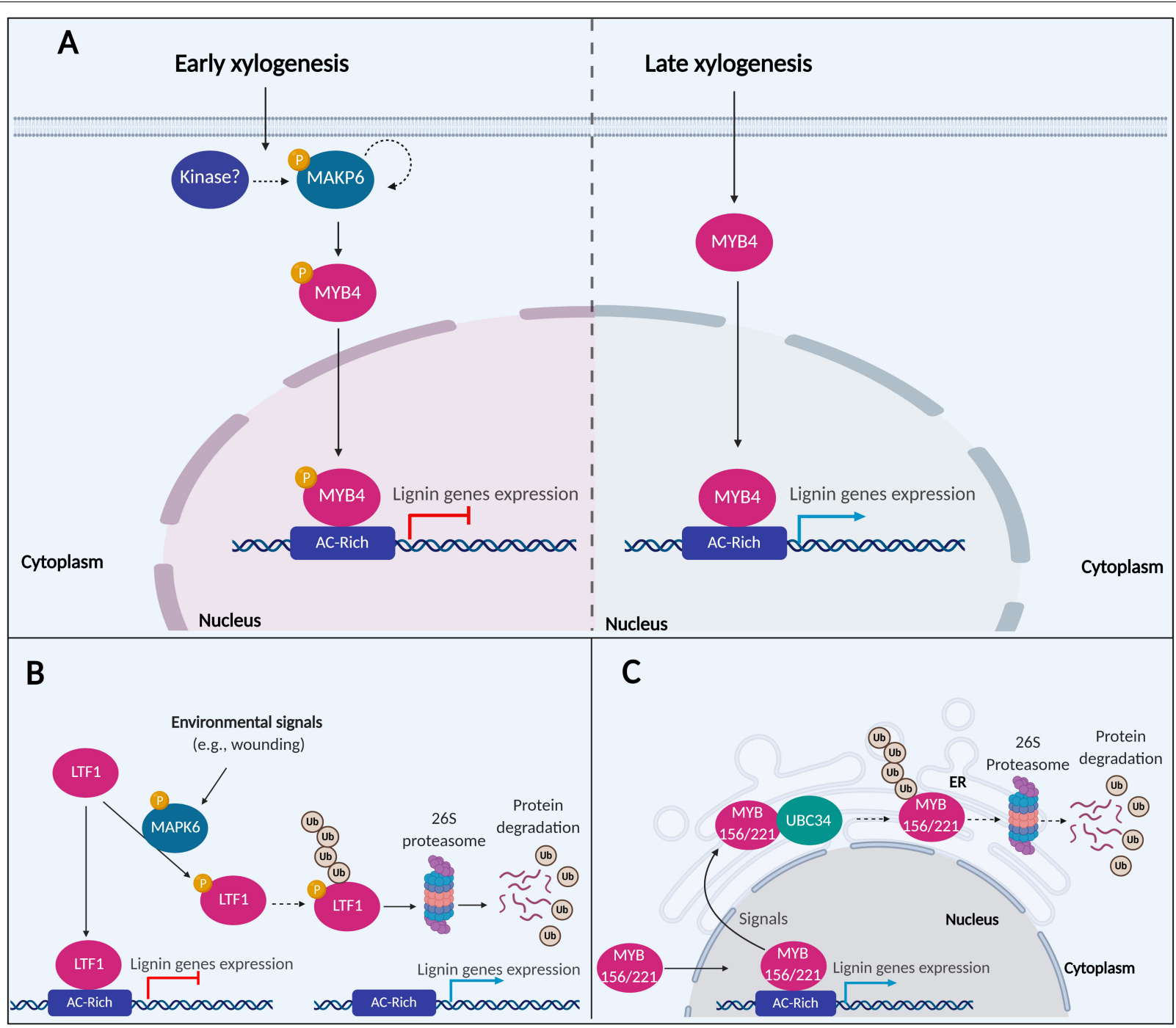

FIGURE 2 | A putative model of MAPK6 regulating the activation of lignin biosynthesis. (A) In the early xylogenesis stage in P. taeda, MAPK6 proteins are autophosphorylated or activated by other kinase-mediated phosphorylation. MAPK6 becomes activated and phosphorylates the TF MYB4. Phosphorylation inactivates MYB4, resulting in the repression of lignin genes. In the late xylogenesis stage, MAPK6 is no longer active, and MYB4 induces the expression of lignin genes (B) In P. trichocarpa, LTF1 is a repressor of lignin genes under normal conditions. After environmental stimuli such as wounding, MAPK6 can interact and phosphorylate LTF1. Phosphorylation destabilizes LTF1 in the cells and promotes its degradation via 26S proteasome and attenuating the repression of lignin genes mediated by LRF1. (C) A putative model of MYB156 and MYB221 regulating the activation of lignin biosynthesis in P. tomentosa. MYB156 and MYB221 are repressors TFs of lignin genes. UBC34 ubiquitin-conjugating enzyme interacts with MYB156 and MYB221 and alters the subcellular localization of the TFs from the nucleus to the ER. The expression of the lignin genes are attenuated either by the TFs trapping into ER or translocation of the MYB156 and MYB221 either traps or degradation via ubiquitin and $26 \mathrm{~S}$ proteasome pathway. The dotted arrows indicated hypothesized events not confirmed experimentally. 
genes, including 4CL. LTF1 mutants in poplar showed elevated transcript expression of lignin genes and increased lignin content. The overexpression of LTF1 in transgenic poplar showed opposite effects, decreasing the expression of lignin genes and reducing the overall lignin content. LTF1 overexpression induced dwarfism in 2-month old poplar (grown in phytotron), but the severity of dwarfism was reduced when the plants reached eight months old (Gui et al., 2019). The protein abundance of LTF1 was decreased significantly in 8-month-old plants. Phosphoproteomic analysis identified two phosphopeptides corresponding to LTF1 in 8 -month old plants. These phosphopeptides were not detected in the 2-month-old LTF1-overexpressed plants. Disruption of the phosphorylation sites in transgenic plants exhibited reduced plant height, stem diameter, and internode length, and reduced lignin deposition in the fiber cells of these plants, and xylem vessel collapse (Gui et al., 2019). Recombinant LTF1 proteins were rapidly degraded when co-incubated with SDX extracts, and the addition of proteasome inhibitor MG-132 re-establishes the initial levels LTF1. On the other hand, even in the absence of MG-132, the levels of LTF1 protein containing mutations in the phosphorylation sites remained stable after the incubation with the SDX extracts.

PdMAPK6 in $P$. deltoides $\times P$. euramericana is a homolog of the Arabidopsis and P. taeda MAPK6, identified by proteinprotein interactions to be associated with LTF1 (Morse et al., 2009; Gui et al., 2019). In vitro phosphorylation assays showed that PdMAPK6 could phosphorylate LTF1 at the Threonine146 and Threonine178 positions, while mutagenesis of these phosphorylation sites prevented LTF1 phosphorylation. A higher level of phosphorylated LTF1 and other PdMAPK6 activated proteins were detected in 8-month old greenhousegrown poplar, compared to 2-month old plants grown in indoor growth chambers. Greenhouse-grown plants are more susceptible to environmental signals like biotic and abiotic stress (Gui et al., 2019), consistent with the functions of MAPK6 in pathogenic response (Andreasson and Ellis, 2010; Bacete et al., 2018). In this context, after mechanical wounding, increased expression of lignin genes and improved lignin deposition were detected in plants overexpressing LTF1. However, these effects were significantly lower in plants overexpressing LTF1 without the phosphorylation sites. Consistently, the expression of Arabidopsis ortholog AtMYB4 is repressed by environmental stimuli, including UV-B and wounding (Jin et al., 2000). These data suggest that LTF1 is a repressor of lignin genes, reducing the lignin content in plant cells. Under environmental stimuli, LTF1 is phosphorylated, decreasing its stability by degradation via $26 \mathrm{~S}$ proteasome (Gui et al., 2019; Figure 2B).

The high peptide sequence identity of PdMAKP6 and PtMAKP6 (84\%) and the similar phosphoregulation of MYB TFs suggest an important role of MAPK in the regulation of lignin biosynthesis in both angiosperm and gymnosperm species. Future studies focusing on MYB phosphorylation by MAPK6 and their interaction with external stimuli would better inform the phosphoregulation of lignin biosynthesis. For instance, is mechanical wounding the only stimulus of LTF1 phosphorylation, or are other signals such as ethylene response, cell wall biosynthesis, and cell remodeling also involved? PTMs are found in diverse classes of proteins, highlighting the regulatory interplay among PTMs as a common strategy to regulate protein functions (Yang, 2005; Nussinov et al., 2012; Lothrop et al., 2013; Pejaver et al., 2014). Other types of PTMs could also be involved in the regulation of MYB TFs. Identifying the upstream regulators of MAPK6 and other wood TFs phosphorylated by MAPK6 are needed to determine the network of regulatory interactions by kinases in the lignification of tree species.

\section{UBIQUITINATION OF PAL}

Ubiquitination is a common regulatory mechanism in all eukaryotes that target proteins for degradation via the $26 \mathrm{~S}$ proteasome, thereby maintaining protein turnover in the cells. The ubiquitin attachment process involves three enzymes: the E1 ubiquitin-activating enzyme, the E2 ubiquitin-conjugating enzyme, and an E3 ubiquitin ligase (Guo and Yang, 2017). Proteins containing a well-conserved 40-50 amino acid F-box domain in the $\mathrm{N}$-terminal region are referred to as F-box proteins that are one part of the ubiquitin E3 ligase complex. F-box proteins are involved in many physiological processes in plants, such as flowering, pathogen defenses, circadian cycle, and phytohormones signaling (Kuroda et al., 2002). PAL enzymes from Arabidopsis (PAL1, PAL2, PAL3, and PAL4) have been shown to interact with Kelch domain-containing F-box (KFB) proteins (KFB01, KFB20, KFB39, and KFB50) (Zhang et al., 2013; Zhang et al., 2015). The F-box domain in KFBs that confers ubiquitin ligase activity is associated with reducing PAL protein abundance by decreasing protein stability (Zhang et al., 2013). Co-overexpression of PALs-GFP and KFBs in Arabidopsis reduced the conversion efficiency of phenylalanine to t-cinnamic acid by up to $80 \%$ (Zhang et al., 2013). More recently, a protein called Small and Glossy Leaves 1 (SAGL1) was identified to be involved in the PTM regulation PAL1. Phylogenetic analysis using 99 Arabidopsis KFB homologous proteins revealed that SAGL1 is closely related to the PAL-regulating KFBs (KFB01, KFB20, KFB39, and KFB50), but located in a separate clade from these KFBs (Yu et al., 2019). SAGL1 can interact and reduce the stability of PAL1, leading to reduced PAL activity for monolignol biosynthesis. Similar to other KFB-mediated PALs regulation, the level of PAL1 was fully restored after MG-132 treatment for proteasome inhibition (Yu et al., 2019).

KFB and SAGL1 have similar regulatory roles in PAL protein stability. Incubation of recombinant PAL in protein extracts of 6-week old Arabidopsis stems led to a significant reduction in the abundance of PAL proteins. The addition of the MG-132 proteasome inhibitor to the assay could maintain PAL protein abundance by preventing ubiquitination-based protein degradation (Zhang et al., 2013). Double and triple mutants in Arabidopsis for the KFB01, KFB20, and KFB50 genes showed an increased amount of PAL proteins, and consequently, more acetyl-bromide lignin in the plant cell walls. Overexpression of KFBs genes, in contrast, caused a 2 to $70 \%$ lignin reduction in the transgenics (Zhang et al., 2013). SAGL1 mutants in Arabidopsis showed a strong purple color in rosette leaves, 
leaf petioles, and inflorescence stems, typically found where high amounts of anthocyanin are accumulated (Nakatsuka and Nishihara, 2010; Yu et al., 2019). Quantification of lignin content in the mature stems of the mutants showed a 2-fold increase in lignin content compared to wild-type. The SAGL1 mutants also had a $60 \%$ increased conversion rate of $\mathrm{L}$-phenylalanine to trans-cinnamate, compared to wild-type. PAL enzyme activity increased in SAGL1 mutants without changing the transcript levels of the PAL genes ( $\mathrm{Yu}$ et al., 2019). Transgenic lines overexpressing SAGL1 showed significant reductions in the conversion rate of L-phenylalanine to trans-cinnamate and lignin content in the mature stems compared to the wild-type (Yu et al., 2019).

The reduced abundance of PAL proteins in Arabidopsis overexpressing KFBS or SAGL1 was not due to reduced transcript levels of $P A L$ genes. In contrast, increased transcript abundance of PALs were observed in the transgenic lines overexpressing KFBs, possibly to compensate for the rapid turnover of PAL proteins in the cells (Zhang et al., 2013). The discrepancy between PAL transcript and protein abundances in the transgenics is indicative of cross-talks between transcriptional regulation and PTM to maintain homeostasis for lignin biosynthesis. PAL mediates the entry step in the phenylpropanoid pathway and is extensively regulated for metabolic control (Zhang and Liu, 2015). In O. sativa, an auxin-responsive Kelch-domain-containing F-box protein (OsFBK1) interacts with cinnamoyl-CoA reductase (OsCCR) to mediate its protein degradation via the proteasome pathway, thereby regulating lignin content in the cell walls (Borah and Khurana, 2018). This suggests that the ubiquitin E3 ligase complex proteins may interact with other lignin biosynthetic enzymes. In $P$. trichocarpa, 337 F-box proteins were identified using a bioinformatics approach (Schumann et al., 2011), suggesting a possible role of these proteins in the regulation of turnover rate in monolignol enzymes. The involvement of this class of protein in regulating lignin biosynthesis and wood formation in tree species remains to be validated.

\section{S-NITROSYLATION AND UBIQUITINATION OF TFS}

TFs containing the NAM/ATAF/CUC (NAC) domain are key regulators of plant development, senescence, SCW formation, and biotic and abiotic stress responses (Olsen et al., 2005; Nakashima et al., 2007; Puranik et al., 2012; Chen X. et al., 2014). Several members of the NAC family of TFs have been identified and characterized in Arabidopsis, rice, soybean, wheat, poplar, and citrus (Puranik et al., 2012). In $O$. sativa, pathogen infection induces the phosphorylation of OsNAC4, leading to its accumulation in the cell nucleus. OsNAC4 is involved in the transregulation of 139 genes and hypersensitive cell death (Kaneda et al., 2009), but its association with SWC biosynthesis has not been reported. Seven NAC genes are highly transcribed during vessel cell differentiation in Arabidopsis (Kubo, 2005). VND7 has been extensively characterized as a transregulator of protoxylem vessel development (Kubo, 2005; Yamaguchi et al., 2010). Repression of VND7 and VND6 using plant repression domain (SRDX) resulted in defects in Arabidopsis growth and vessel formation, mediated by repressed central metaxylem vessel formation and central protoxylem vessel formation (Kubo, 2005). Induced expression of VND7 accelerated vessel cell differentiation in all cell types encompassing cotyledon, leaf, hypocotyl, and root cells (Yamaguchi et al., 2010; Kawabe et al., 2018).

The knockout of S-nitrosoglutathione reductase 1 (GSNOR1) in transgenic Arabidopsis expressing VND7 resulted in mutant seedlings lacking xylem vessel differentiation (mutant "suppressor of ectopic vessel cell differentiation induced by VND7" or seiv) (Yamaguchi et al., 2010; Kawabe et al., 2018). Indeed, the inducible-VND7 expression in the seiv mutants did not show any evidence of cell wall deposition. These mutant seedlings showed a dwarf phenotype, suggesting that the GSNOR1 gene may be involved in plant growth and development, in addition to regulating VND7 for xylem trans-differentiation (Kawabe et al., 2018). VND7 directly or indirectly regulates the expression of genes involved in SCW formation during xylem vessel differentiation, encompassing the biosynthesis of cellulose, hemicellulose, and lignin, and programed cell death (Yamaguchi et al., 2011). The expression of these VND7-downstream genes is induced by VND7 overexpression, and suppressed in seiv mutants, demonstrating that the knockout of GSNOR1 disrupts VND7-mediated regulation (Kawabe et al., 2018). Biotin switch assays showed that the VND7 was S-nitrosylated in vitro, and amino acid substitution of two Cysteine264 and Cysteine320 abolished the S-nitrosylation signals. The presence of S-nitrosoglutathione reduced VND7 activity in Arabidopsis protoplasts. VND7 activity was reduced when either Cysteine264 or Cysteine320 were substituted for tryptophan, which mimics a constitutive S-nitrosylated isoform of VND7. Thus, S-nitrosylation plays an essential role in negatively regulating VND7 activity for vessel development. However, replacing the Cysteine264 or Cysteine320 for a serine residue, which mimics a non-S-nitrosylated form of VND7, also caused a reduction in the transactivation activity of VND7 (Kawabe et al., 2018). Cysteine residues in plant proteins are modified by different types of PTMs in addition to S-nitrosylation, including S-sulfenylation, S-glutathionylation, sulfinylation, sulfonylation, and the formation of intra-/intermolecular disulfide bridges (Waszczak et al., 2015; Kawabe et al., 2018). Therefore, other PTMs may be regulating VND7 and causing the non-S-nitrosylated isoform of VND7 to reduce transactivation activity in protoplasts (Kawabe et al., 2018).

NAC-domain proteins have been demonstrated to interact with many cellular components involved in different cellular processes (Yamaguchi et al., 2008), making them a potential target for protein modifications. For instance, the RING domain protein SINA of Arabidopsis 5 (SINAT5) interacts with NAC1 and promotes its degradation via the ubiquitin/26S proteasome pathway (Olsen et al., 2005). Similarly, VND7 
is regulated by $26 \mathrm{~S}$ proteasome-mediated degradation (Yamaguchi et al., 2008). Protein extracts from transformed tobacco BY-2 cells expressing VND7 had low levels of detected VND7 protein after $24 \mathrm{~h}$, while the addition of proteasome inhibitor increased the abundance of VND7 (Yamaguchi et al., 2008).

Although herbaceous plants such as Arabidopsis have a limited secondary growth compared to tree species, some similarities may be found in the general organization of their tissues (Chaffey et al., 2002; Demura and Fukuda, 2007; Zhong et al., 2010b). Repeated removal of inflorescent stems in Arabidopsis can induce some secondary xylem production that has been used in developmental studies of secondary growth (Oh et al., 2003). Searching for NAC TFs in the $P$. trichocarpa genome, Zhong et al. (2010b) identified 16 homologous genes of Arabidopsis VND, NST, and SMB involved in secondary wall biosynthesis or xylem differentiation. These poplar genes were named PtrWNDs for "wood-associated NAC domain transcription factors" (Zhong et al., 2010b; Ohtani et al., 2011). Of the 16 genes, 12 showed a high level of transcript expression in vessel and fiber cells of developing woody tissue. Moreover, they were localized in the nucleus of plant cells and exhibited transactivation activities, consistent with their putative function as TFs (Zhong et al., 2010b). Expression of PtrWNDs in Arabidopsis snd1 (nst3) nst 1 double mutants rescued the pendent inflorescence stem phenotype and restored stem strength and deposition of secondary walls in interfascicular fibers. Overexpression of PtrWND2B and PtrWND6B (sub members of the $S M B$ and $V N D$ class of $\mathrm{TF}$, respectively) in Arabidopsis increased the transcript expression of cellulose synthases (CesA4, CesA7, and CesA8), xylan biosynthetic genes (FRA8, IRX8, and IRX9), and lignin biosynthetic genes (4CL1 and CCoAOMT1) (Zhong et al., 2010b; Ohtani et al., 2011). PtrWND2B and PtrWND6B also transactivated wood-associated TFs involved in the biosynthesis of lignin, hemicelluloses, and cellulose (Zhong et al., 2010b). Overexpression of 12 PtVNS/PtrWND in $P$. trichocarpa caused SCW formation surrounding the transgenics' vascular tissue, similarly to which was seen for the induced expression of VND7 in Arabidopsis and poplar epidermal cells (Ohtani et al., 2011). Expression of VND7 in poplar induced the expression of many genes related to SCW formation, including enzyme-encoding genes and TFs (Ohtani et al., 2011). Taken together, NAC TFs in Arabidopsis and $P$. trichocarpa are functionally important for SCW formation (Zhong et al., 2010a; Ohtani et al., 2011). Orthologous proteins are typically conserved in their PTMs, and the level of conservation is higher in closely related species (Remmerie et al., 2011; Venne et al., 2014). PTMs identified for Arabidopsis NAC TFs, such as S-nitrosylation and ubiquitination, may also occur in PtrWNDs in the regulation of wood formation.

In P. tomentosa, PtoMYB156 and PtoMYB221 from the subgroup 4 of the R2R3-MYB TF family work as transrepressors of lignin genes (Zheng et al., 2019). Overexpression of PtoMYB156 dramatically retarded plant growth and reduced secondary xylem formation, while thickening of SCWs in the vascular stem tissue was observed in PtoMYB156 knockout plants (Yang et al., 2017). The regulation of PtoMYB156 and PtoMYB221 is mediated by interactions with an E2 ubiquitin-conjugating enzyme 34 (PtoUBC34), which translocate the TFs to the ER (Zheng et al., 2019). In mesophyll protoplasts of $P$. tomentosa overexpressing PtoMYB156 and PtoMYB221, the TFs are localized in the cell nucleus and could suppress the transcript expression of target genes in the absence of PtoUBC34. When PtoUBC34 is co-expressed in the same system, PtoMYB156 and PtoMYB221 are translocated to the ER in a dose-depend way, and their repression activity is reduced (Zheng et al., 2019). Whether the reduced repression activity of PtoMYB156 and PtoMYB221 is due to the trapping of these TFs in the ER or degradation via the 26S proteasome pathway remains unknown (Figure 2C). Lignin biosynthesis responds to various developmental and environmental signals such as light, sugar content, circadian rhythms, plant hormones, and wounding, where these signals are converted to a physiological process by hierarchical transcriptional regulation of lignin pathway genes (Zheng et al., 2019). The translocation to cellular compartments or possibly ubiquitination and degradation via the $26 \mathrm{~S}$ proteasome pathway may be a pivotal point in regulating the activity of these TFs. Future studies will help to clarify the crosstalking of lignin biosynthesis and other physiological processes (Zheng et al., 2019).

\section{GLYCOSYLATION IN PEROXIDASES}

Class III peroxidases are present as large multigenic families in all land plants (Mathé et al., 2010). Generally, this class of proteins is heme-containing enzymes, which may oxidize a wide range of substrates using hydrogen peroxide as a reduction agent. Peroxidases are associated with multiple cellular processes, encompassing primary and secondary metabolism, hormone catabolism, pathogen defense, phenol oxidation, cross-linking of cell wall-structural proteins, and polysaccharides, and lignin polymerization (Christensen et al., 1998). As aforementioned, peroxidases oxidize the monolignols in the plant cell walls to free radicals for conjugating to the growing lignin polymer (Li et al., 2014). The oxidation reaction mediated by lignin peroxidases is characterized by a three-step cycle involving a two-electron enzyme oxidation: FeIII $+\mathrm{H}_{2} \mathrm{O}_{2} \rightarrow \mathrm{CoI}+\mathrm{H}_{2} \mathrm{O}$ (k1); followed by two one-electron reductions: (i) $\mathrm{CoI}+\mathrm{RH}$ $\rightarrow \mathrm{CoII}+\mathrm{R}$; (k2) and (ii) $\mathrm{CoII}+\mathrm{RH} \rightarrow \mathrm{FeIII}+\mathrm{R}$; $(k 3)$, where FeIII, CoI, CoII, and $\mathrm{RH}$ are the native enzyme, compound I, compound II, and the monolignol, respectively (Gabaldón et al., 2006).

Most of the peroxidases are glycosylated, and the carbohydrate content may constitute up to $25 \%$ of the protein (Christensen et al., 1998; Veitch, 2004) Glycosylation sites are normally found in the sequence motif asn-x-ser/thr (where $\mathrm{x}$ means any amino acid residue), and they occur within surface turns or loop regions connecting helices (Veitch, 2004). Six anionic stem peroxidases (PXP1-6) were isolated from xylem tissues of P. trichocarpa, and all were heavily glycosylated, exhibiting $\mathrm{N}$-glucosamine and ten 
putative glycosylation sites in their structures (Christensen et al., 1998). Similarly, two isoforms of ZePrx, where one was fully glycosylated (ZePrx 34.70) and another partially glycosylated (ZePrx 33.44) were identified in Zinnia elegans suspensions cells (Gabaldón et al., 2005; Gabaldón et al., 2007). The two isoforms of this protein showed different biochemical properties. For instance, the $k 1$ (CoI formation constant - which monitors the reactivity of the enzyme with $\mathrm{H}_{2} \mathrm{O}_{2}$ ) was higher in the ZePrx 34.70 compared to ZePrx 33.44 in the presence of either $p$-coumaryl alcohol or coniferyl alcohol substrates. On the other hand, the opposite effects were observed when coniferaldehyde, sinapyl alcohol, and sinapaldehyde were used as substrates. Moreover, the $k 3$, (CoII reduction constant - which monitors the ability of the oxidized form of the enzyme, CoII, to oxidize phenolics) was higher for ZePrx 33.44 comparing to the ZePrx 34.70 in the presence of coniferyl alcohol. Again, the opposite effects were observed in the presence of sinapyl alcohol, where the reactivity was higher for the ZePrx 34.70 (Gabaldón et al., 2006). The different reactivity rates suggest that glycans modulate the catalytic activity of the peroxidases (Gabaldón et al., 2006). Since multiple glycosylation sites are found in these enzymes, the different glycosylation patterns may determine the substrate specificity for monolignol oxidation, leading to changes in the composition of the lignin. Glycosylation may have a role beyond the regulation of peroxidase substrate specificity. Protein folding and stabilization, and protein-cell wall interactions have also been predicted as putative functions of this PTM in peroxidases (Schuller et al., 1996; Gabaldón et al., 2007; Cesarino et al., 2012).

\section{FINAL CONSIDERATIONS}

Despite the technical challenges in the detection and characterization of PTMs in vivo, recent discoveries have provided insights into their importance in regulating lignin biosynthesis and wood formation. The lignin biosynthetic pathway is complex and controlled by many external and developmental stimuli. The complexity of the regulations suggests that TFs, monolignol enzymes, and peroxidases could respond rapidly and specifically to different types of stimuli in the modulation of lignin biosynthesis. Phosphorylation (e.g., PAL2 and AldOMT2) and ubiquitination (e.g., PALs and CCR) of monolignol enzymes decrease the enzymatic activity and protein stability (Allwood et al., 1999; Zhang et al., 2013; Wang J. P. et al., 2015; Borah and Khurana, 2018). More comprehensive identification of PTMs associated with lignin biosynthesis will further improve our understanding of the spatiotemporal regulation of the pathway, and how such regulations coordinately affect the properties of the cell walls. MAPKs mediated MYB4 and LTF1 phosphorylation (Morse et al., 2009; Gui et al., 2019) have provided valuable mechanistic insights on how PTM modulates the SCW but the complete signaling pathway and the interactions between MAPKs and other MAPK-cascade components remain to be elucidated. Further studies focusing on how developmental and environmental signals trigger the MAPK-cascade to activate or repress TFs regulating lignin biosynthesis would be valuable to determine the network of regulatory interactions that control phenotypic alterations.

Protein degradation via $26 \mathrm{~S}$ proteasome (e.g., VND7, LTF1, and possibly MYB156 and MYB221) plays an essential role in regulating TFs for lignin biosynthesis (Kawabe et al., 2018; Gui et al., 2019; Zheng et al., 2019). However, there are knowledge gaps to be filled for a better understand of how TF protein turnover is modulated by PTMs. Ubiquitination, which is related to the $26 \mathrm{~S}$ proteasome pathway, remains to be identified in LTF1, MYB156, and MYB221. Moreover, the combinatorial effects of multiple PTMs and their coordinated control of lignin biosynthesis remain poorly understood. For example, the glycosylation of lignin peroxidases (e.g., ZePrx) controls the enzyme substrate specificity (Gabaldón et al., 2006), and further studies may elucidate how multiple glycosylations, and glycosylation together with other PTMs may combinatorially affect lignin composition and structure. Moreover, the relationship between VND7 S-nitrosylation and other types of cysteine modifications in the transregulation of SCW genes is unknown (Kawabe et al., 2018). One PTM can influence the modification of other types of PTMs, which can result in a broad variation of possible proteoforms (Friso and van Wijk, 2015). The combinatorial effect of multiple PTMs and cross-talk between PTMs in the same protein, or on different proteins within complexes, is crucial to defining the interrelationships of multiple PTMs in lignin biosynthesis.

Much of the early work on plant PTMs have focused on model organisms such as Arabidopsis. These PTM in woody species, particularly in the regulation of lignin biosynthesis has remained largely unknown. Due to the large variation in lignin content, composition, and structure between Arabidopsis and perennial woody species, the extent of similarity or difference in the SCWassociated PTMs across species should be evaluated. Systematic co-expression analysis of SCW protein abundances with PTM enzymes during SDX formation, or genome-scale protein interactomics could reveal putative PTM regulatory interactions controlling lignin biosynthesis. The resulting interactions may guide future studies to verify whether these PTMs occur in vitro and in vivo and how they regulate lignin biosynthesis and wood formation. Comprehensive identification of PTMs in trees will be crucial to understanding the transduction of complex regulatory information that mediates wood formation.

\section{AUTHOR CONTRIBUTIONS}

DS and JW conceptualized and literary reviewed and wrote the manuscript.

\section{FUNDING}

This work was supported by the United States Department of Agriculture (Grant\# 2017-06529) and the NC State University Chancellor's Innovation Fund (Grant\# 190549MA). 


\section{REFERENCES}

Adams-Phillips, L., Briggs, A. G., and Bent, A. F. (2010). Disruption of poly(ADPribosyl)ation mechanisms alters responses of Arabidopsis to biotic stress. Plant Physiol. 152, 267-280. doi: 10.1104/pp.109.148049

Akamatsu, A., Wong, H. L., Fujiwara, M., Okuda, J., Nishide, K., Uno, K., et al. (2013). An OsCEBiP/OsCERK1-OsRacGEF1-OsRac1 module is an essential early component of chitin-induced rice immunity. Cell Host Microbe 13, 465476. doi: 10.1016/j.chom.2013.03.007

Allwood, E. G., Davies, D. R., Gerrish, C., Ellis, B. E., and Bolwell, G. P. (1999), Phosphorylation of phenylalanine ammonia-lyase: evidence for a novel protein kinase and identification of the phosphorylated residue. FEBS Lett. 457, 47-52. doi: 10.1016/S0014-5793(99)00998-9

Andreasson, E., and Ellis, B. (2010). Convergence and specificity in the Arabidopsis MAPK nexus. Plant Sci. 15, 106-113. doi: 10.1016/j.tplants.2009.12.001

Bacete, L., Mélida, H., Miedes, E., and Molina, A. (2018). Plant cell wall-mediated immunity: cell wall changes trigger disease resistance responses. Plant J. 93, 614-636. doi: 10.1111/tpj.13807

Bell-Lelong, D. A., Cusumano, J. C., Meyer, K., and Chapple, C. (1997). Cinnamate-4-hydroxylase expression in Arabidopsis. Regulation in response to development and the environment. Plant Physiol. 113, 729-738. doi: 10.1104/ pp.113.3.729

Beltrao, P., Bork, P., Krogan, N. J., and Noort, V. (2013). Evolution and functional cross-talk of protein post-translational modifications. Mol. Syst. Biol. 9:714. doi: $10.1002 / \mathrm{msb} .201304521$

Borah, P., and Khurana, J. P. (2018). The OsFBK1 E3 ligase subunit affects anther and root secondary cell wall thickenings by mediating turnover of a cinnamoyl-CoA reductase. Plant Physiol. 176, 2148-2165. doi: 10.1104/pp.17. 01733

Catala, R., Ouyang, J., Abreu, I. A., Hu, Y., Seo, H., Zhang, X., et al. (2007). The Arabidopsis E3 SUMO ligase SIZ1 regulates plant growth and drought responses. Plant Cell 19, 2952-2966. doi: 10.1105/tpc.106.04 9981

Cesarino, I., Araújo, P., Sampaio Mayer, J. L., Paes Leme, A. F., and Mazzafera, P. (2012). Enzymatic activity and proteomic profile of class III peroxidases during sugarcane stem development. Plant Physiol. Biochem. 55, 66-76. doi: 10.1016/j.plaphy.2012.03.014

Chaffey, N., Cholewa, E., Regan, S., and Sundberg, B. (2002). Secondary xylem development in Arabidopsis: a model for wood formation. Physiol. Plant. 114, 594-600. doi: 10.1034/j.1399-3054.2002.1140413.x

Chen, H.-C., Li, Q., Shuford, C. M., Liu, J., Muddiman, D. C., Sederoff, R. R., et al. (2011). Membrane protein complexes catalyze both 4- and 3-hydroxylation of cinnamic acid derivatives in monolignol biosynthesis. Proc. Natl. Acad. Sci. U.S.A. 108, 21253-21258. doi: 10.1073/pnas.1116416109

Chen, H.-C., Song, J., Wang, J. P., Lin, Y.-C., Ducoste, J., Shuford, C. M., et al. (2014). Systems biology of lignin biosynthesis in Populus trichocarpa: heteromeric 4-coumaric acid:coenzyme A ligase protein complex formation, regulation, and numerical modeling. Plant Cell 26, 876-893. doi: 10.1105/tpc. 113.119685

Chen, X., Wang, Y., Lv, B., Li, J., Luo, L., Lu, S., et al. (2014). The NAC family transcription factor OsNAP confers abiotic stress response through the ABA pathway. Plant Cell Physiol. 55, 604-619. doi: 10.1093/pcp/pct204

Chen, H.-C., Wang, J. P., Liu, H., Li, H., Lin, Y.-C. J., and Shi, R. (2019). Hierarchical transcription factor and chromatin binding network for wood formation in Populus trichocarpa. Plant Cell 31, 602-626. doi: 10.1105/tpc.18. 00620

Chen, Z., Zheng, Z., Huang, J., Lai, Z., and Fan, B. (2009). Biosynthesis of salicylic acid in plants. Plant Signal. Behav. 4, 493-496. doi: 10.4161/psb.4.6.8392

Cheng, S.-H., Sheen, J., Gerrish, C., and Bolwell, G. P. (2001). Molecular identification of phenylalanine ammonia-lyase as a substrate of a specific constitutively active Arabidopsis CDPK expressed in maize protoplasts. FEBS Lett. 503, 185-188. doi: 10.1016/S0014-5793(01)02732-6

Christensen, J. H., Bauw, G., Gjesing Welinder, K., Van Montagu, M., and Boerjan, W. (1998). Purification and characterization of peroxidases correlated with lignification in poplar xylem. Plant Physiol. 118, 125-135. doi: 10.1104/pp.118. 1.125

Demura, T., and Fukuda, H. (2007). Transcriptional regulation in wood formation. Trends Plant Sci. 12, 64-70. doi: 10.1016/j.tplants.2006.12.006
Friso, G., and van Wijk, K. J. (2015). Update: post-translational protein modifications in plant metabolism. Plant Physiol. 169, 1469-1487. doi: 10.1104/ pp. 15.01378

Fromm, J. (2013). "Xylem development in trees: from cambial divisions to mature wood cells," in cellular Aspects of Wood Formation, ed. J. Fromm (Berlin: Springer Berlin Heidelberg), 3-39.

Gabaldón, C., Gómez-Ros, L. V., Núñez-Flores, M. J. L., Esteban-Carrasco, A., and Barceló, A. R. (2007). Post-translational modifications of the basic peroxidase isoenzyme from Zinnia elegans. Plant Mol. Biol. 65, 43-61. doi: 10.1007/s11103007-9197-0

Gabaldón, C., López-Serrano, M., Pedreño, M. A., and Barceló, A. R. (2005). Cloning and molecular characterization of the basic peroxidase isoenzyme from Zinnia elegans, an enzyme involved in lignin biosynthesis. Plant Physiol. 139, 1138-1154. doi: 10.1104/pp.105.069674

Gabaldón, C., López-Serrano, M., Pomar, F., Merino, F., Cuello, J., Pedreño, M. A., et al. (2006). Characterization of the last step of lignin biosynthesis in Zinnia elegans suspension cell cultures. FEBS Lett. 580, 4311-4316. doi: 10.1016/j. febslet.2006.06.088

Gao, Z. M., Wang, X. C., Peng, Z. H., Zheng, B., and Liu, Q. (2012). Characterization and primary functional analysis of phenylalanine ammonialyase gene from Phyllostachys edulis. Plant Cell Rep. 31, 1345-1356. doi: 10. 1007/s00299-012-1253-9

Goicoechea, M., Lacombe, E., Legay, S., Mihaljevic, S., Rech, P., Jauneau, A., et al. (2005). EgMYB2, a new transcriptional activator from Eucalyptus xylem, regulates secondary cell wall formation and lignin biosynthesis: EgMYB2, a regulator of lignification. Plant J. 43, 553-567. doi: 10.1111/j.1365-313X.2005. 02480.x

Gou, M., Ran, X., Martin, D. W., and Liu, C.-J. (2018). The scaffold proteins of lignin biosynthetic cytochrome P450 enzymes. Nat. Plants 4, 299-310. doi: 10.1038/s41477-018-0142-9

Gui, J., Luo, L., Zhong, Y., Sun, J., Umezawa, T., and Li, L. (2019). Phosphorylation of LTF1, an MYB transcription factor in Populus, acts as a sensory switch regulating lignin biosynthesis in wood cells. Mol. Plant 12, 1325-1337. doi: 10.1016/j.molp.2019.05.008

Guo, T., and Yang, X. (2017). Ubiquitination and its function in defense pathways. Eur. J. Biomed. Res. 3, 1-4. doi: 10.18088/ejbmr.3.2.2017

Hamann, T. (2012). Plant cell wall integrity maintenance as an essential component of biotic stress response mechanisms. Front. Plant Sci. 3:77. doi: 10.3389/fpls. 2012.00077

Hatton, D., Sablowski, R., Yung, M. H., Smith, C., Schuch, W., and Bevan, M. (1995). Two classes of cis sequences contribute to tissue-specific expression of a PAL2 promoter in transgenic tobacco. Plant J. 7, 859-876. doi: 10.1046/j.1365313X.1995.07060859.x

Hauffe, K. D., Lee, S. P., Subramaniam, R., and Douglas, C. J. (1993). Combinatorial interactions between positive and negative cis-acting elements control spatial patterns of 4CL-1 expression in transgenic tobacco. Plant J. 4, 235-253. doi: 10.1046/j.1365-313X.1993.04020235.x

Jin, H., Cominelli, E., Bailey, P., Parr, A., Mehrtens, F., Jones, J., et al. (2000). Transcriptional repression by AtMYB4 controls production of UV-protecting sunscreens in Arabidopsis. EMBO J. 19, 6150-6161. doi: 10.1093/emboj/19.22. 6150

Jin, H., and Martin, C. (1999). Multifunctionality and diversity within the plant MYB-gene family. Plant Mol. Biol. 41, 577-585. doi: 10.1023/A:1006319732410

Jones, D. H. (1984). Phenylalanine ammonia-lyase: regulation of its induction, and its role in plant development. Phytochemistry 23, 1349-1359. doi: 10.1016/ S0031-9422(00)80465-3

Joos, H.-J., and Hahlbrock, K. (1992). Phenylalanine ammonia-lyase in potato (Solanum tuberosum L.). Genomic complexity, structural comparison of two selected genes and modes of expression. Eur. J. Biochem. 204, 621-629. doi: 10.1111/j.1432-1033.1992.tb16675.x

Kaneda, T., Taga, Y., Takai, R., Iwano, M., Matsui, H., Takayama, S., et al. (2009). The transcription factor OsNAC4 is a key positive regulator of plant hypersensitive cell death. EMBO J. 28, 926-936. doi: 10.1038/emboj.20 09.39

Karpinska, B., Karlsson, M., Srivastava, M., Stenberg, A., Schrader, J., Sterky, F., et al. (2004). MYB transcription factors are differentially expressed and regulated during secondary vascular tissue development in hybrid aspen. Plant Mol. Biol. 56, 255-270. doi: 10.1007/s11103-004-3354-5 
Kawabe, H., Ohtani, M., Kurata, T., Sakamoto, T., and Demura, T. (2018). Protein s-nitrosylation regulates xylem vessel cell differentiation in Arabidopsis. Plant Cell Physiol. 59, 17-29. doi: 10.1093/pcp/pcx151

Kawasaki, T., Koita, H., Nakatsubo, T., Hasegawa, K., Wakabayashi, K., Takahashi, H., et al. (2006). Cinnamoyl-CoA reductase, a key enzyme in lignin biosynthesis, is an effector of small GTPase Rac in defense signaling in rice. Proc. Natl. Acad. Sci. U.S.A. 103, 230-235. doi: 10.1073/pnas.0509875103

Kim, S.-H., Oikawa, T., Kyozuka, J., Wong, H. L., Umemura, K., Kishi-Kaboshi, M., et al. (2012). The bHLH Rac immunityl (RAI1) is activated by OsRac1 via OsMAPK3 and OsMAPK6 in rice immunity. Plant Cell Physiol. 53, 740-754. doi: $10.1093 / \mathrm{pcp} / \mathrm{pcs} 033$

Kubo, M. (2005). Transcription switches for protoxylem and metaxylem vessel formation. Genes Dev. 19, 1855-1860. doi: 10.1101/gad.1331305

Kuroda, H., Takahashi, N., Shimada, H., Seki, M., Shinozaki, K., and Matsui, M. (2002). Classification and expression analysis of Arabidopsis F-Box-containing protein genes. Plant Cell Physiol. 43, 1073-1085. doi: 10.1093/pcp/pcf151

Lacombe, E., Van Doorsselaere, J., Boerjan, W., Boudet, A. M., and GrimaPettenati, J. (2000). Characterization of cis-elements required for vascular expression of the cinnamoyl-CoA reductase gene and for protein-DNA complex formation. Plant J. 23, 663-676. doi: 10.1046/j.1365-313x.2000. 00838. $\mathrm{x}$

Lauvergeat, V., Rech, P., Jauneau, A., Guez, C., Coutos-Thevenot, P., and GrimaPettenati, J. (2002). The vascular expression pattern directed by the Eucalyptus gunnii cinnamyl alcohol dehydrogenase EgCAD2 promoter is conserved among woody and herbaceous plant species. Plant Mol. Biol. 50, 497-509.

Legay, S., Sivadon, P., Blervacq, A.-S., Pavy, N., Baghdady, A., Tremblay, L., et al. (2010). EgMYB1, an R2R3 MYB transcription factor from eucalyptus negatively regulates secondary cell wall formation in Arabidopsis and poplar. New Phytol. 188, 774-786. doi: 10.1111/j.1469-8137.2010.03432.x

Leyva, A., Liang, X., Pintor-Toro, J. A., Dixon, R. A., and Lamb, C. J. (1992). Ciselement combinations determine phenylalanine ammonia-lyase gene tissuespecific expression patterns. Plant Cell 4, 263-271. doi: 10.1105/tpc.4.3.263

Li, C., Wang, X., Ran, L., Tian, Q., Fan, D., and Luo, K. (2015). PtoMYB92 is a transcriptional activator of the lignin biosynthetic pathway during secondary cell wall formation in Populus tomentosa. Plant Cell Physiol. 56, 2436-2446. doi: $10.1093 / p c p / p c v 157$

Li, Q., Lin, Y.-C., Sun, Y.-H., Song, J., Chen, H., Zhang, X.-H., et al. (2012). Splice variant of the SND1 transcription factor is a dominant negative of SND1 members and their regulation in Populus trichocarpa. Proc. Natl. Acad. Sci. U.S.A. 109, 14699-14704. doi: 10.1073/pnas.1212977109

Li, Q., Min, D., Wang, J. P.-Y., Peszlen, I., Horvath, L., Horvath, B., et al. (2011). Down-regulation of glycosyltransferase 8D genes in Populus trichocarpa caused reduced mechanical strength and xylan content in wood. Tree Physiol. 31, 226-236. doi: 10.1093/treephys/tpr008

Li, Q., Song, J., Peng, S., Wang, J. P., Qu, G.-Z., Sederoff, R. R., et al. (2014). Plant biotechnology for lignocellulosic biofuel production. Plant Biotechnol. J. 12, 1174-1192. doi: $10.1111 /$ pbi.12273

Lin, Y.-C., Li, W., Sun, Y.-H., Kumari, S., Wei, H., Li, Q., et al. (2013). SND1 transcription factor-directed quantitative functional hierarchical genetic regulatory network in wood formation in Populus trichocarpa. Plant Cell 25, 4324-4341. doi: 10.1105/tpc.113.117697

Logemann, E., Parniske, M., and Hahlbrock, K. (1995). Modes of expression and common structural features of the complete phenylalanine ammonia-lyase gene family in parsley. Proc. Natl. Acad. Sci. U.S.A. 92, 5905-5909. doi: 10.1073/pnas. 92.13.5905

Lois, R., Dietrich, A., Hahlbrock, K., and Schulz, W. (1989). A phenylalanine ammonia-lyase gene from parsley: structure, regulation and identification of elicitor and light responsive cis-acting elements. EMBO J. 8, 1641-1648. doi: 10.1002/j.1460-2075.1989.tb03554.x

Lothrop, A. P., Torres, M. P., and Fuchs, S. M. (2013). Deciphering posttranslational modification codes. FEBS Lett. 587, 1247-1257. doi: 10.1016/j. febslet.2013.01.047

Mann, M., and Jensen, O. N. (2003). Proteomic analysis of post-translational modifications. Nat. Biotechnol. 21, 255-261. doi: 10.1038/nbt0303-255

Mathé, C., Barre, A., Jourda, C., and Dunand, C. (2010). Evolution and expression of class III peroxidases. Arch. Biochem. Biophys. 500, 58-65. doi: 10.1016/j.abb. 2010.04.007
Mauriat, M., Leplé, J.-C., Claverol, S., Bartholomé, J., Negroni, L., Richet, N., et al. (2015). Quantitative proteomic and phosphoproteomic approaches for deciphering the signaling pathway for tension wood formation in poplar. J. Proteome Res. 14, 3188-3203. doi: 10.1021/acs.jproteome.5b0 0140

Mazzucotelli, E., Mastrangelo, A. M., Crosatti, C., Guerra, D., Stanca, A. M., and Cattivelli, L. (2008). Abiotic stress response in plants: when post-transcriptional and post-translational regulations control transcription. Plant Sci. 174, 420431. doi: 10.1016/j.plantsci.2008.02.005

Miura, K., and Hasegawa, P. M. (2010). Sumoylation and other ubiquitin-like post-translational modifications in plants. Trends Cell Biol. 20, 223-232. doi: 10.1016/j.tcb.2010.01.007

Morse, A. M., Whetten, R. W., Dubos, C., and Campbell, M. M. (2009). Posttranslational modification of an R2R3-MYB transcription factor by a MAP kinase during xylem development. New Phytol. 183, 1001-1013. doi: 10.1111/ j.1469-8137.2009.02900.x

Nakashima, K., Tran, L.-S. P., Van Nguyen, D., Fujita, M., Maruyama, K., Todaka, D., et al. (2007). Functional analysis of a NAC-type transcription factor OsNAC6 involved in abiotic and biotic stress-responsive gene expression in rice: rice OsNAC6 functions in stress responses. Plant J. 51, 617-630. doi: 10.1111/j.1365-313X.2007.03168.x

Nakatsuka, T., and Nishihara, M. (2010). UDP-glucose:3-deoxyanthocyanidin 5O-glucosyltransferase from Sinningia cardinalis. Planta 232, 384-392. doi: 10. 1007/s00425-010-1175-0

Nasir, F., Tian, L., Chang, C., Li, X., Gao, Y., Tran, L.-S. P., et al. (2018). Current understanding of pattern-triggered immunity and hormone-mediated defense in rice (Oryza sativa) in response to Magnaporthe oryzae infection. Semin. Cell Dev. Biol. 83, 95-105. doi: 10.1016/j.semcdb.2017.10.020

Nørregaard Jensen, O. (2004). Modification-specific proteomics: characterization of post-translational modifications by mass spectrometry. Curr. Opin. Chem. Biol. 8, 33-41. doi: 10.1016/j.cbpa.2003.12.009

Nühse, T. S., Peck, S. C., Hirt, H., and Boller, T. (2000). Microbial Elicitors Induce Activation and Dual Phosphorylation of the Arabidopsis thaliana MAPK6. J. Biol. Chem. 275, 7521-7526. doi: 10.1074/jbc.275.11.7521

Nussinov, R., Tsai, C.-J., Xin, F., and Radivojac, P. (2012). Allosteric posttranslational modification codes. Trends Biochem. Sci. 37, 447-455. doi: 10. 1016/j.tibs.2012.07.001

Oh, S., Park, S., and Han, K.-H. (2003). Transcriptional regulation of secondary growth in Arabidopsis thaliana. J. Exp. Bot. 54, 2709-2722. doi: 10.1093/jxb/ $\operatorname{erg} 304$

Ohtani, M., Nishikubo, N., Xu, B., Yamaguchi, M., Mitsuda, N., Goué, N., et al. (2011). A NAC domain protein family contributing to the regulation of wood formation in poplar: NAC domain protein family regulates wood formation. Plant J. 67, 499-512. doi: 10.1111/j.1365-313X.2011.04614.x

Olsen, A. N., Ernst, H. A., Leggio, L. L., and Skriver, K. (2005). NAC transcription factors: structurally distinct, functionally diverse. Trends Plant Sci. 10, 79-87. doi: $10.1016 /$ j.tplants.2004.12.010

Patzlaff, A., McInnis, S., Courtenay, A., Surman, C., Newman, L. J., Smith, C., et al. (2003a). Characterisation of a pine MYB that regulates lignification. Plant J. 36, 743-754. doi: 10.1046/j.1365-313X.2003.01916.x

Patzlaff, A., Newman, L. J., Dubos, C., Whetten, R. W., Smith, C., McInnis, S., et al. (2003b). Characterisation of PtMYB1, an R2R3-MYB from pine xylem. Plant Mol. Biol. 53, 597-608. doi: 10.1023/B:PLAN.0000019066.07933.d6

Pejaver, V., Hsu, W.-L., Xin, F., Dunker, A. K., Uversky, V. N., and Radivojac, P. (2014). The structural and functional signatures of proteins that undergo multiple events of post-translational modification: structural and functional signatures of PTM crosstalk. Protein Sci. 23, 1077-1093. doi: 10.1002/pro.2494

Puranik, S., Sahu, P. P., Srivastava, P. S., and Prasad, M. (2012). NAC proteins: regulation and role in stress tolerance. Trends Plant Sci. 17, 369-381. doi: 10.1016/j.tplants.2012.02.004

Rasmussen, S., and Dixon, R. A. (1999). Transgene-mediated and elicitorinduced perturbation of metabolic channeling at the entry point into the phenylpropanoid pathway. Plant Cell 11, 1537-1551. doi: 10.1105/tpc.11.8. 1537

Remmerie, N., De Vijlder, T., Laukens, K., Dang, T. H., Lemière, F., Mertens, I., et al. (2011). Next generation functional proteomics in non-model plants: a survey on techniques and applications for the analysis of protein complexes and 
post-translational modifications. Phytochemistry 72, 1192-1218. doi: 10.1016/j. phytochem.2011.01.003

Ritter, H., and Schulz, G. E. (2004). Structural basis for the entrance into the phenylpropanoid metabolism catalyzed by phenylalanine ammonia-lyase. Plant Cell 16, 3426-3436. doi: 10.1105/tpc.104.025288

Romeis, T. (2001). Calcium-dependent protein kinases play an essential role in a plant defence response. EMBO J. 20, 5556-5567. doi: 10.1093/emboj/20.20. 5556

Schuller, D. J., Ban, N., van Huystee, R. B., McPherson, A., and Poulos, T. L. (1996). The crystal structure of peanut peroxidase. Structure 4, 311-321. doi: 10.1016/S0969-2126(96)00035-4

Schulz, P., Herde, M., and Romeis, T. (2013). Calcium-dependent protein kinases: hubs in plant stress signaling and development. Plant Physiol. 163, 523-530. doi: $10.1104 /$ pp.113.222539

Schumann, N., Navarro-Quezada, A., Ullrich, K., Kuhl, C., and Quint, M. (2011). Molecular evolution and selection patterns of plant F-Box proteins with C-terminal kelch repeats. Plant Physiol. 155, 835-850. doi: 10.1104/pp.110. 166579

Séguin, A., Laible, G., Leyva, A., Dixon, R. A., and Lamb, C. J. (1997). Characterization of a gene encoding a DNA-binding protein that interacts in vitro with vascular specific cis elements of the phenylalanine ammonia-lyase promoter. Plant Mol. Biol. 35, 281-291. doi: 10.1023/A:1005853404242

Seifert, G. J., and Blaukopf, C. (2010). Irritable walls: the plant extracellular matrix and signaling. Plant Physiol. 153, 467-478. doi: 10.1104/pp.110.153940

Shi, R., Shuford, C. M., Wang, J. P., Sun, Y.-H., Yang, Z., Chen, H.-C., et al. (2013). Regulation of phenylalanine ammonia-lyase (PAL) gene family in wood forming tissue of Populus trichocarpa. Planta 238, 487-497. doi: 10.1007/ s00425-013-1905-1

Shoresh, M., and Harman, G. E. (2008). The molecular basis of shoot responses of maize seedlings to Trichoderma harzianum T22 inoculation of the root: a proteomic approach. Plant Physiol. 147, 2147-2163. doi: 10.1104/pp.108. 123810

Shuford, C. M., Li, Q., Sun, Y.-H., Chen, H.-C., Wang, J., Shi, R., et al. (2012). Comprehensive quantification of monolignol-pathway enzymes in Populus trichocarpa by protein cleavage isotope dilution mass spectrometry. J. Proteome Res. 11, 3390-3404. doi: 10.1021/pr300205a

Solecka, D. (1997). Role of phenylpropanoid compounds in plant responses to different stress factors. Acta Physiol. Plant. 19, 257-268. doi: 10.1007/s11738997-0001-1

Speicher, T., Li, P., and Wallace, I. (2018). Phosphoregulation of the plant cellulose synthase complex and cellulose synthase-like proteins. Plants 7:52. doi: 10.3390/ plants7030052

Spoel, S. H. (2018). Orchestrating the proteome with post-translational modifications. J. Exp. Bot. 69, 4499-4503. doi: 10.1093/jxb/ery295

Stulemeijer, I. J. E., and Joosten, M. H. A. J. (2008). Post-translational modification of host proteins in pathogen-triggered defence signalling in plants. Mol. Plant Pathol. 9, 545-560. doi: 10.1111/j.1364-3703.2008.00468.x

Tian, Q., Wang, X., Li, C., Lu, W., Yang, L., Jiang, Y., et al. (2013). Functional characterization of the poplar R2R3-MYB transcription factor PtoMYB216 involved in the regulation of lignin biosynthesis during wood formation. PLoS One 8:e76369. doi: 10.1371/journal.pone.0076369

Veitch, N. C. (2004). Structural determinants of plant peroxidase function. Phytochem. Rev. 3, 3-18. doi: 10.1023/B:PHYT.0000047799.17604.94

Venne, A. S., Kollipara, L., and Zahedi, R. P. (2014). The next level of complexity: Crosstalk of posttranslational modifications. Proteomics 14, 513-524. doi: 10. 1002/pmic.201300344

Wang, G.-F., and Balint-Kurti, P. J. (2016). Maize homologs of CCoAOMT and HCT, two key enzymes in lignin biosynthesis, form complexes with the NLR Rp1 protein to modulate the defense response. Plant Physiol. 171, 2166-2177. doi: 10.1104/pp.16.00224

Wang, G.H., He, Y., Strauch, R., Olukolu, B., Nielsen, D., Li, X., et al. (2015). Maize homologs of HCT, a key enzyme in lignin biosynthesis, bind the NLR Rp1 proteins to modulate the defense response. Plant Physiol. 169, 2230-2243. doi: 10.1104/pp.15.00703

Wang, J. P., Chuang, L., Loziuk, P. L., Chen, H., Lin, Y.-C., Shi, R., et al. (2015). Phosphorylation is an on/off switch for 5-hydroxyconiferaldehyde O methyltransferase activity in poplar monolignol biosynthesis. Proc. Natl. Acad. Sci. U.S.A. 112, 8481-8486. doi: 10.1073/pnas.1510473112
Wang, S., Li, E., Porth, I., Chen, J.-G., Mansfield, S. D., and Douglas, C. J. (2015). Regulation of secondary cell wall biosynthesis by poplar R2R3 MYB transcription factor PtrMYB152 in Arabidopsis. Sci. Rep. 4:5054. doi: 10.1038/ srep05054

Wang, J. P., Liu, B., Sun, Y., Chiang, V. L., and Sederoff, R. R. (2019a). Enzymeenzyme interactions in monolignol biosynthesis. Front. Plant Sci. 9:1942. doi: 10.3389/fpls.2018.01942

Wang, J. P., Matthews, M. L., Naik, P. P., Williams, C. M., Ducoste, J. J., Sederoff, R. R., et al. (2019b). Flux modeling for monolignol biosynthesis. Curr. Opin. Biotechnol. 56, 187-192. doi: 10.1016/j.copbio.2018. 12.003

Wang, J. P., Matthews, M. L., Williams, C. M., Shi, R., Yang, C., Tunlaya-Anukit, S., et al. (2018). Improving wood properties for wood utilization through multiomics integration in lignin biosynthesis. Nat. Commun. 9:1579. doi: 10.1038/ s41467-018-03863-Z

Wang, J. P., Naik, P. P., Chen, H.-C., Shi, R., Lin, C.-Y., Liu, J., et al. (2014). Complete proteomic-based enzyme reaction and inhibition kinetics reveal how monolignol biosynthetic enzyme families affect metabolic flux and lignin in Populus trichocarpa. Plant Cell 26, 894-914. doi: 10.1105/tpc.113. 120881

Waszczak, C., Akter, S., Jacques, S., Huang, J., Messens, J., and Van Breusegem, F. (2015). Oxidative post-translational modifications of cysteine residues in plant signal transduction. J. Exp. Bot. 66, 2923-2934. doi: 10.1093/jxb/ erv084

Yamaguchi, M., Goué, N., Igarashi, H., Ohtani, M., Nakano, Y., Mortimer, J. C., et al. (2010). VASCULAR-RELATED NAC-DOMAIN6 and VASCULARRELATED NAC-DOMAIN7 effectively induce transdifferentiation into xylem vessel elements under control of an induction system. Plant Physiol. 153, 906-914. doi: 10.1104/pp.110.154013

Yamaguchi, M., Kubo, M., Fukuda, H., and Demura, T. (2008). VASCULARRELATED NAC-DOMAIN7 is involved in the differentiation of all types of xylem vessels in Arabidopsis roots and shoots. Plant J. 55, 652-664. doi: 10.1111/ j.1365-313X.2008.03533.x

Yamaguchi, M., Mitsuda, N., Ohtani, M., Ohme-Takagi, M., Kato, K., and Demura, T. (2011). VASCULAR-RELATED NAC-DOMAIN 7 directly regulates the expression of a broad range of genes for xylem vessel formation: direct target genes of VND7. Plant J. 66, 579-590. doi: 10.1111/j.1365-313X.2011. 04514.x

Yan, S., and Dong, X. (2014). Perception of the plant immune signal salicylic acid. Curr. Opin. Plant Biol. 20, 64-68. doi: 10.1016/j.pbi.2014. 04.006

Yang, L., Zhao, X., Ran, L., Li, C., Fan, D., and Luo, K. (2017). PtoMYB156 is involved in negative regulation of phenylpropanoid metabolism and secondary cell wall biosynthesis during wood formation in poplar. Sci. Rep. 7:41209. doi: $10.1038 /$ srep41209

Yang, L., Zhao, X., Yang, F., Fan, D., Jiang, Y., and Luo, K. (2016). PtrWRKY19, a novel WRKY transcription factor, contributes to the regulation of pith secondary wall formation in Populus trichocarpa. Sci. Rep 6:18643. doi: 10.1038/ srep 18643

Yang, X.-J. (2005). Multisite protein modification and intramolecular signaling. Oncogene 24, 1653-1662. doi: 10.1038/sj.onc.1208173

Ye, Z.-H., and Zhong, R. (2015). Molecular control of wood formation in trees. J. Exp. Bot. 66, 4119-4131. doi: 10.1093/jxb/erv081

Yu, S., Kim, H., Yun, D.-J., Suh, M. C., and Lee, B. (2019). Post-translational and transcriptional regulation of phenylpropanoid biosynthesis pathway by kelch repeat F-box protein SAGL1. Plant Mol. Biol. 99, 135-148. doi: 10.1007/s11103018-0808-8

Zhang, X., Gou, M., Guo, C., Yang, H., and Liu, C.-J. (2015). Down-regulation of kelch domain-containing F-Box protein in Arabidopsis enhances the production of (poly)phenols and tolerance to ultraviolet radiation. Plant Physiol. 167, 337-350. doi: 10.1104/pp.114.249136

Zhang, X., Gou, M., and Liu, C.-J. (2013). Arabidopsis kelch repeat F-Box proteins regulate phenylpropanoid biosynthesis via controlling the turnover of phenylalanine ammonia-lyase. Plant Cell 25, 4994-5010. doi: 10.1105/tpc.113. 119644

Zhang, X., and Liu, C.-J. (2015). Multifaceted regulations of gateway enzyme phenylalanine ammonia-lyase in the biosynthesis of phenylpropanoids. Mol. Plant 8, 17-27. doi: 10.1016/j.molp.2014.11.001 
Zheng, L., Chen, Y., Ding, D., Zhou, Y., Ding, L., Wei, J., et al. (2019). Endoplasmic reticulum-localized UBC34 interaction with lignin repressors MYB221 and MYB156 regulates the transactivity of the transcription factors in Populus tomentosa. BMC Plant Biol. 19:97. doi: 10.1186/s12870-019-1697-y

Zhong, R., Lee, C., and Ye, Z.-H. (2010a). Evolutionary conservation of the transcriptional network regulating secondary cell wall biosynthesis. Trends Plant Sci. 15, 625-632. doi: 10.1016/j.tplants.2010.08.007

Zhong, R., Lee, C., and Ye, Z.-H. (2010b). Functional characterization of poplar wood-associated NAC domain transcription factors. Plant Physiol. 152, 10441055. doi: 10.1104/pp.109.148270
Conflict of Interest: The authors declare that the research was conducted in the absence of any commercial or financial relationships that could be construed as a potential conflict of interest.

Copyright (c) 2020 Sulis and Wang. This is an open-access article distributed under the terms of the Creative Commons Attribution License (CC BY). The use, distribution or reproduction in other forums is permitted, provided the original author(s) and the copyright owner(s) are credited and that the original publication in this journal is cited, in accordance with accepted academic practice. No use, distribution or reproduction is permitted which does not comply with these terms. 